\title{
My 2,000 best films: parallel phenotyping of Dictyostelium development Gareth Bloomfield ${ }^{* \dagger}$ and Robert R Kay*
} Addresses: *MRC Laboratory of Molecular Biology, Hills Road, Cambridge CB2 OQH, UK. ${ }^{\top}$ The Wellcome Trust Sanger Institute, Hinxton
CB10 1SA, UK.

Correspondence: Robert R Kay. Email: rrk@mrc-lmb.cam.ac.uk

Published: 23 July 2007

Genome Biology 2007, 8:220 (doi:10.1 I 86/gb-2007-8-7-220)

The electronic version of this article is the complete one and can be found online at http://genomebiology.com/2007/8/7/220

(C) 2007 BioMed Central Ltd

\begin{abstract}
A new study has used parallel filming to record the development of 2,000 Dictyostelium mutants, and clustered them into related groups using morphological staging and wavelet analysis of aggregation patterns.
\end{abstract}

Genetic screens have driven progress in molecular biology from its earliest days. Think of the fine structure of the gene as studied in phage $\mathrm{T} 4$ [1], the delineation of cell-cycle genes in yeast [2] and the identification of genes underlying development in Drosophila [3]. Such screens always require ingenuity of inception but, especially in metazoa, they also require skill and patience to carry out. Screens for mutants in the fly eye have revealed many important aspects of growth factor signaling, but they required a breeding program to produce randomly mutated individuals and then skilled microscopic observation of the compound eyes to identify aberrant patterns of differentiation. It is therefore not surprising that it has been difficult to increase the productivity of such screens to match that of genome sequencing and other genomic methods.

This situation is slowly changing. For instance, RNA interference (RNAi) or directed gene deletion can replace traditional mutagenesis, with the advantage that saturation of the genome is approached directly rather than asymptotically and the identity of the mutated genes is known from the outset. Coupled to this, automated microscopy and image analysis allows enormous amounts of data to be accumulated and analyzed from mutant individuals. For example, near-complete collections of gene-deletion mutants have been amassed and studied in parallel in budding yeast [4,5], gene expression has been systematically perturbed by RNAi and the consequences captured by time-lapse filming in Caenorhabditis elegans [6], and defects in mitotic progression in cultured carcinoma cells have been screened for using RNAi and automated image analysis [7].

Traditional genetic screens succeeded best where they could pick out mutants affecting the process of interest and reject others. But if sufficient data can be gathered, and appropriately clustered, then it may be possible to perform a single comprehensive screen from which many different classes of mutants can be recognized. In a paper published in Genome Biology [8], Sawai and colleagues report a screen for developmental mutants of the social amoeba Dictyostelium discoideum, based on parallel time-lapse filming, which moves in this direction.

\section{The particularities of Dictyostelium}

Dictyostelium brings particular biological advantages to such an enterprise, due to its ease of culture and genetic manipulation $[9,10]$. Its genome is compact and has been fully sequenced, with around 12,500 genes [11]. Positioned among the crown group of eukaryotes, it is notable for having many genes in common with metazoans that have been lost from yeast. An alternative set of cell biological problems is addressable compared with yeast, including cell motility and chemotaxis, phagocytosis and extensive intercellular communication. Its position on the border between unicellular and multicellular life also makes 
Dictyostelium particularly interesting for studies on the evolution of multicellularity and the consequent appearance of altruistic cell types (in this case dead stalk cells), which also brings the necessity of avoiding cheating [12].

In the wild, Dictyostelium grows as individual amoebae that feed on bacteria, but domesticated versions can grow in defined media. In both cases, depletion of food provokes a remarkable social behavior: the previously individualistic cells organize themselves into multicellular aggregates by chemotaxis guided by cyclic AMP. After a few hours of starvation, a few cells spontaneously release cyclic AMP, which diffuses towards their immediate neighbors. These respond by chemotaxis towards the cyclic AMP source - the aggregation center - and themselves release a pulse of cyclic AMP, which diffuses towards and attracts more distant cells, and so on. The periodic waves of cyclic AMP traversing the field of amoebae can be visualized microscopically by the cellular responses of rounding and elongation that they elicit as they pass over responsive cells. The truly multicellular aggregates thus formed then undergo a programmed series of morphological changes, including directed migration across the substratum to a suitable place to fruit, and ultimately the formation of a fruiting body or sorocarp, consisting of a slender stalk supporting a mass of spores. The spores can then be dispersed to locations where growth can recommence.

\section{Parallel time-lapse filming of Dictyostelium development}

Sawai and colleagues [8] used a robotic gantry camera system to film the development of up to 100 mutant clones in parallel. The aggregation waves of early development could be visualized after image processing and were characterized using wavelet analysis [13]. This analytical tool uses mathematical functions to capture important periodic features within data. Wavelet transforms follow similar principles to Fourier transforms, with the important difference that they are particularly suited to local, evolving patterns in data. Later stages of development were scored morphologically, with qualitative phenotypes fitted into a coarse quantitative scale. The whole dataset could then be clustered to give a number of groupings of mutants that make biological sense. For instance, strains mutant in genes in a histidine kinase phosphorelay system that regulates intracellular cyclic AMP levels clustered together, as did strains mutant in a number of genes required at the initiation of development.

Sawai et al. [8] report that using this method they found that about $4 \%$ of the mutants analyzed were found to have a clear developmental defect, although they grew normally as individual amoebae. This makes one wonder about the other 96\%, which showed no phenotypic defects in this assay. These mutants were created by insertion of a single plasmid anywhere in the genome [14], although with some bias towards insertion into coding sequences. Many insertions may be in unimportant intergenic regions and literally have no phenotype; other insertions will be in redundant genes. Some mutants will affect growth, but not in the conditions used; others may have subtle developmental phenotypes undetected in this analysis; and yet others may affect processes that were not assayed by this screen. For instance, Dictyostelium can form resistant cells called aspidocytes in response to toxic attack [15], and forms macrocysts as part of its sexual cycle [16]. It also appears well equipped for ecological interaction by production of polyketide signals and antibiotics, but none of these aspects of its life-style would be expected to be elicited in the conditions studied by Sawai et al. [8].

\section{Expanding the horizon}

Apart from widening the range of experimental conditions examined, the application of parallel filming can be expanded in several directions. First, and most obvious, it will be desirable to saturate the protein-coding potential of the genome with mutations. Genes nonessential for normal growth can be examined for possible developmental functions in loss-of-function mutants. The analysis system of Sawai et al. [8] clearly has the capacity to do this, but the production of mutants will become limiting as saturation is approached, and it will be necessary to switch from the current random plasmid-insertion methods to directed gene knockout to play the end-game efficiently. The remaining essential genes can, in principle, be studied using partial loss-of-function alleles created using RNAi to knock down expression [17].

The dynamics of the cyclic AMP oscillator in aggregation have been modeled previously $[13,18]$, but the wealth of quantitative data now available from the study of Sawai et al. [8], and the identification of further genes affecting this oscillator should enable these models to be put on a more realistic biological basis. Connection of the film data [8] to the Dictyostelium database [19], which is under way, will make this information readily available.

Finally, the parallel-filming approach is ideal for screening drugs and inhibitors. One bottleneck in the procedure of Sawai et al. [8] is the growth and preparation for development of so many strains. For drug screening, it would be possible to use just a single strain - the wild type or a sensitized mutant, mutated in the process of interest to render them more sensitive to additional mutations in the same process - and then use the technique to determine the effects of adding known drugs and chemical libraries in parallel. Similar clustering and classification methods to those used by Sawai et al. [8] could be applied to the data and genetic phenotypes matched to chemical ones, thus giving a direct insight into the target of the drug. 


\section{References}

I. Benzer S: On the topology of the genetic fine structure. Proc Natl Acad Sci USA 1959, 45: 1607-1620.

2. Hartwell LH, Culotti J, Pringle JR, Reid BJ: Genetic control of the cell division cycle in yeast. Science 1974, 183:46-51.

3. Nusslein-Volhard C, Wieschaus E: Mutations affecting segment number and polarity in Drosophila. Nature 1980, 287:795-80I.

4. Winzeler EA, Shoemaker DD, Astromoff A, Liang H, Anderson K, Andre B, Bangham R, Benito R, Boeke JD, Bussey H, et al.: Functional characterization of the $S$. cerevisiae genome by gene deletion and parallel analysis. Science 1999, 285:90I-906.

5. Giaever G, Chu AM, Ni L, Connelly C, Riles L, Veronneau S, Dow S, Lucau-Danila A, Anderson K, Andre B, et al.: Functional profiling of the Saccharomyces cerevisiae genome. Nature 2002, 4I 8:387391.

6. Kamath RS, Fraser AG, Dong Y, Poulin G, Durbin R, Gotta M, Kanapin A, Le Bot N, Moreno S, Sohrmann M, et al.: Systematic functional analysis of the Caenorhabditis elegans genome using RNAi. Nature 2003, 42 I:231-237.

7. Moffat J, Grueneberg DA, Yang X, Kim SY, Kloepfer AM, Hinkle G, Piqani B, Eisenhaure TM, Luo B, Grenier JK, et al:: A lentiviral RNAi library for human and mouse genes applied to an arrayed viral high-content screen. Cell 2006, I 24: | 283-1298.

8. Sawai S, Guan X-J, Kuspa A, Cox EC: High-throughput analysis of spatio-temporal dynamics in Dictyostelium. Genome Biol 2007, 8:R I 44.

9. Kay RR, Williams JG: The Dictyostelium genome project: an invitation to species hopping. Trends Genet 1999, 15:294-297.

10. Kessin RH: Dictyostelium. Cambridge: Cambridge University Press; 2001.

II. Eichinger L, Pachebat JA, Glockner G, Rajandream MA, Sucgang R, Berriman M, Song J, Olsen R, Szafranski K, Xu Q, et al.: The genome of the social amoeba Dictyostelium discoideum. Nature 2005, 435:43-57.

12. Foster KR, Shaulsky G, Strassmann JE, Queller DC, Thompson CR: Pleiotropy as a mechanism to stabilize cooperation. Nature 2004, $431: 693-696$.

13. Sawai S, Thomason PA, Cox EC: An autoregulatory circuit for long-range self-organization in Dictyostelium cell populations. Nature 2005, 433:323-326.

14. Kuspa A, Loomis WF: Tagging developmental genes in Dictyostelium by restriction enzyme-mediated integration of plasmid DNA. Proc Natl Acad Sci USA 1992, 89:8803-8807.

15. Serafimidis I, Bloomfield G, Skelton J, Ivens A, Kay RR: A new environmentally resistant cell type from Dictyostelium. Microbiology 2007, 153:619-630.

16. Urushihara H, Muramoto T: Genes involved in Dictyostelium discoideum sexual reproduction. Eur J Cell Biol 2006, 85:96I-968.

17. Martens H, Novotny J, Oberstrass J, Steck TL, Postlethwait P, Nellen W: RNAi in Dictyostelium: the role of RNA-directed RNA polymerases and double-stranded RNase. Mol Biol Cell 2002, 13:445-453.

18. Martiel JL, Goldbeter A: Autonomous chaotic behaviour of the slime mould Dictyostelium discoideum predicted by a model for cyclic AMP signaling. Nature 1985, 3 1 3:590-592.

19. dictyBase [http://dictybase.org] 